\title{
Lazy Set-Sharing Analysis
}

\author{
Xuan $\mathrm{Li}^{1}$, Andy King ${ }^{2}$, Lunjin $\mathrm{Lu}^{1}$ \\ 1 Oakland University Rochester, MI 48309, USA \\ $\{\mathrm{x} 2 \mathrm{li}, 12 \mathrm{lu}\} @$ oakland.edu \\ 2 University of Kent, Canterbury, UK \\ a.m.king@kent.ac.uk
}

\begin{abstract}
Sharing analysis is widely deployed in the optimisation, specialisation and parallelisation of logic programs. Each abstract unification operation over the classic Jacobs and Langen domain involves the calculation of a closure operation that has exponential worst-case complexity. This paper explores a new tactic for improving performance: laziness. The idea is to compute partial sharing information eagerly and recover full sharing information lazily. The net result is an analysis that runs in a fraction of the time of the classic analysis and yet has comparable precision.
\end{abstract}

\section{Introduction}

Sharing analysis is one of the most well-studied analyses within logic programming. Two variables are said to share if they are bound to terms that contain a common variable; otherwise they are independent. Independence information has many applications in logic programming that include occurs-check reduction [24, 10], automatic parallelisation [11] and finite-tree analysis [2]. Set-sharing analysis $[17,18]$, as opposed to pair-sharing analysis [24], has particularly attracted much attention. This is because researchers have been repeatedly drawn to the algebraic properties of the domain since these offer tantalising opportunities for implementation $[3,7,8,12]$.

The reoccurring problem with the set-sharing domain is the closure under union operation that lies at the heart of the abstract unification (amgu) operation [18]. A sharing abstraction is constructed from a set of sharing groups each of which is a set of program variables. Closure under union operation repeatedly unions together sets of sharing groups, drawn from a given sharing abstraction, until no new sharing group can be obtained. This operation is exponential, hence the interest in different, and possibly more tractable, encodings of set-sharing [7, 8]. One approach to curbing the problem of closure is to schedule the solving of a sequence of equations so as to first apply amgu to equations that involve ground terms [21]. To this end, Muthukumar and Hermenegildo [22] detail an queueing/dequeueing mechanism for maximally propagating groundness among systems of equations. This tactic exploits the commutativity of the amgu operation $[13,21][$ Lemma 62]. A refinement of this idea is to schedule the amgu operations using an estimate of the cost of a closure. The amgu operations are applied in 
order of increasing closure cost and then widening is invoked [25, 21] [Section 4.3] when the application of a closure becomes prohibitively expensive. To support widening, the set-sharing domain has been extended [25, 21][Section 4.3] from a set of sharing groups, to a pair of sets of sharing groups. A sharing group in the first component (that is called a clique [25]) is then reinterpreted as representing all sharing groups that are contained within it.

This paper addresses the question of whether closure calculation is actually required at all. This paper shows that cliques can be reinterpreted as pending closure operations. Under this reinterpretation, the complexity of amgu can be reduced to a quadratic time operation on sharing groups and a constant time operation on cliques. If necessary, a classic set-sharing abstraction can be recovered using the cliques without incurring a precision loss by applying a closure operation for the groups that are relevant to each clique. Quite apart from being rather surprising, this reinterpretation of cliques can avoid computing closures all together in some circumstances. Firstly, if one clique is contained within another, then the smaller clique is redundant as is its associated closure operation. Secondly, if a clique contains those variables that appear in a call, then it can be passed from one procedure to another without applying closure. Thirdly, if closure is prohibitively expensive, then it can be retained as clique, albeit incurring a possible loss of precision in the projection and merge operations. This leads to an analysis that is parameterised by a cost threshold $k$ : a clique is only created if the resulting number of sharing groups exceed $k$. The resulting analysis is polynomial and benchmarking suggests that it realises a good tradeoff between precision and efficiency.

This paper is organised as follows. Section 2 contains basic concepts and recalls the Sharing domain. Section 3 informally introduces the motivation via examples. Sections 4-7 present the abstract domain and abstract operators in details. Section 8 discusses an experimental implementation. Section 9 discusses related work and section 10 concludes.

\section{Preliminaries}

Let $\mathcal{V}$ denote a denumerable universe of variables. Let $\operatorname{var}(o)$ be the set of variables in the syntactic object $o$. If $S$ is a set then $|S|$ is its cardinality and $\wp(S)$ is the powerset of $S$. The (classic) set-sharing domain Sharing is defined by Sharing $=\{S \in \wp(\wp(\mathcal{V})) \mid \emptyset \in S\}$. We call a set of variables $G \subseteq \mathcal{V}$ a sharing group and a set $S \in$ Sharing a sharing abstraction. The restriction $G \uparrow V$ is defined by $G \uparrow V=G \cap V$ where $V \subseteq \mathcal{V}$. Moreover, if $S \in$ Sharing then $S \uparrow V$ is defined by $S \uparrow V=\{G \uparrow V \mid G \in S\}$. The function $\max : \wp(\wp(\mathcal{V})) \rightarrow \wp(\wp(\mathcal{V}))$ is defined by $\max (S)=\{G \in S \mid \forall H \in S . G \subseteq H \rightarrow G=H\}$.

The most important operator in Sharing is the abstract unification operator $a m g u_{x=t}$ where $x$ is a variable and $t$ is a term not containing $x$. The operator $a m g u_{x=t}$ is defined in terms of three auxiliary operations. The closure under union of a sharing abstraction $S$, denoted by $S^{*}$, is the smallest superset of $S$ satisfying if $X \in S^{*}$ and $Y \in S^{*}$ then $X \cup Y \in S^{*}$. The set of sharing groups in 
$S$ that are relevant to a syntactic object $o$ is $\operatorname{rel}(o, S)=\{G \in S \mid \operatorname{var}(o) \cap G \neq$ $\emptyset\}$. We say that a sharing abstraction $S \in$ Sharing is relevant to a term $t$ iff $\operatorname{rel}(t, S) \neq \emptyset$. The cross union of two sharing abstractions $S_{1}$ and $S_{2}$ is defined by $S_{1} \uplus S_{2}=\left\{G \cup H \mid G \in S_{1} \wedge H \in S_{2}\right\}$. The following definition of the abstract unification is adapted from [8] that is equivalent to classic definition in $[17,18]$.

Definition 1. $\operatorname{amgu}_{x=t}(S)=S \backslash \operatorname{rel}(x, S) \backslash \operatorname{rel}(t, S) \cup(\operatorname{rel}(x, S) \uplus \operatorname{rel}(t, S))^{*}$ where $\backslash$ is the set minus operator.

The set of all clique-sets $C L[25]$ is defined by $C L=\wp(\{G \in \wp(\mathcal{V}) \mid G \neq \emptyset\})$. A clique is an element of a clique-set. In this paper, a clique is the abstraction of an equation of the $e$ where $e$ takes the form $x=t$.

\section{Motivation Example}

This section informally introduces the basic idea using several examples. We demonstrate how to use cliques and part of sharing abstraction to represent the whole sharing abstraction.

Example 1. Suppose $S=\left\{\emptyset,\left\{X_{1}\right\},\left\{X_{2}\right\},\left\{X_{3}\right\},\left\{X_{4}\right\},\left\{X_{5}\right\},\left\{X_{6}\right\},\left\{X_{7}\right\}\right\}$ and equation $e$ is $X_{1}=f\left(X_{2}, X_{3}, X_{4}, X_{5}, X_{6}, X_{7}\right)$. If we apply the classical abstract unification $a m g u$, we obtain

$$
\operatorname{amgu}_{e}(S)=\{\emptyset\} \cup\left\{\begin{array}{l}
\left\{X_{1}, X_{2}\right\},\left\{X_{1}, X_{3}\right\},\left\{X_{1}, X_{4}\right\}, \\
\left\{X_{1}, X_{5}\right\},\left\{X_{1}, X_{6}\right\},\left\{X_{1}, X_{7}\right\}
\end{array}\right\}^{*}
$$

There are 63 groups in $\operatorname{amgu}_{e}(S) \backslash\{\emptyset\}$ in all. Note that we only list 6 groups instead of enumerating all 63 groups for the sake of saving space. We shall refer to these 6 groups as the basis of the 63 groups because the whole closure (the 63 groups) can be recovered from the basis (the 6 groups) by applying closure under union operator to the basis. In other words, we can use the basis to represent the whole closure. The intuition behind the analysis is to record which groups participate in the closure under union and avoid computing closure under union operation until it is called for. We record which groups participate in closure under union by keeping a record of the set of variables that are contained within the equation $e$. Moreover, we only consider the equations that contain at least one variable and thus we can use a clique [25] to represent these variable sets. Therefore we use a pair $\langle E, S\rangle \in C L \times$ Sharing to represent a sharing abstraction: the first component is the set of cliques; the second component is the partially computed sharing information. In this way, we can avoid closure under union operation during analysis.

Example 2. Continuing with example 1, the state of sharing after abstract unification is expressed as

$$
\left\langle\begin{array}{l}
\left\{\left\{X_{1}, X_{2}, X_{3}, X_{4}, X_{5}, X_{6}, X_{7}\right\}\right\}, \\
\left\{\emptyset,\left\{X_{1}, X_{2}\right\},\left\{X_{1}, X_{3}\right\},\left\{X_{1}, X_{4}\right\},\left\{X_{1}, X_{5}\right\},\left\{X_{1}, X_{6}\right\},\left\{X_{1}, X_{7}\right\}\right\}
\end{array}\right\rangle
$$

In this example, this part of sharing information is $S \backslash \operatorname{rel}(x=t, S) \cup \operatorname{rel}(x, S) \uplus$ $\operatorname{rel}(t, S)$. We will show that this is not a coincident in the later sections. 
When we need to recover full sharing information, we can just apply the closure under union to those groups that are relevant to each clique.

Example 3. Continuing with example 2, there is only one clique in first components. If we denote $\left\{X_{1}, X_{2}, X_{3}, X_{4}, X_{5}, X_{6}, X_{7}\right\}$ by $e$ and $\left\{\emptyset,\left\{X_{1}, X_{2}\right\},\left\{X_{1}, X_{3}\right\}\right.$, $\left.\left\{X_{1}, X_{4}\right\},\left\{X_{1}, X_{5}\right\},\left\{X_{1}, X_{6}\right\},\left\{X_{1}, X_{7}\right\}\right\}$ by $S$, then the full sharing information is obtained by

$$
S \cup \operatorname{rel}(e, S)^{*}=\{\emptyset\} \cup\left\{\begin{array}{l}
\left\{X_{1}, X_{2}\right\},\left\{X_{1}, X_{3}\right\},\left\{X_{1}, X_{4}\right\}, \\
\left\{X_{1}, X_{5}\right\},\left\{X_{1}, X_{6}\right\},\left\{X_{1}, X_{7}\right\}
\end{array}\right\}^{*}
$$

which coincides with the sharing information derived in example 1.

If there is more than one clique, then sharing is recovered by considering each clique in turn.

Example 4. Consider $\left\langle\left\{e_{1}, e_{2}\right\}, S\right\rangle$ where $e_{1}=\{X, Y\}, e_{2}=\{Y, Z\}$ and $S=$ $\{\emptyset,\{X\},\{Y\},\{Z\}\}$. First, applying $e_{1}$, we obtain $S_{1}=S \cup \operatorname{rel}\left(e_{1}, S\right)^{*}=\{\emptyset,\{X\}$, $\{Y\},\{Z\},\{X, Y\}\}$. Next applying $e_{2}$, we have $S_{1} \cup \operatorname{rel}\left(e_{2}, S_{1}\right)^{*}=\{\emptyset,\{X\},\{Y\}$, $\{Z\},\{X, Y\},\{Y, Z\},\{X, Y, Z\}\}$. In fact, as is shown latter, the order in which the cliques are consider is incidental.

Although we call closure under union operator when we recover full sharing information, we only apply it on demand. Even if the client wants to get full sharing information at every program point, a lazy evaluation strategy is still better because we avoid redundant computation during analysis. For an instance, when clique $e_{1}$ is a subset of $e_{2}$, we can disregard $e_{1}$ because the effect of $e_{1}$ is subsumed by that of $e_{2}$. Moreover, the number of groups involved in least fixed point computation is much less than the number of groups involved in classical Sharing because only part of closure is involved.

In summary, we only compute part of sharing information at each program point during analysis and can recover full sharing information on demand after analysis. As a result, the closure under union operator is avoided for abstract unification. In following sections, we will present this new domain formally.

\section{Abstract Domain}

Reducing the input data size and improving the efficiency of operators over the domain are two ways to improve the efficiency of an analysis. Recall definition 1:

$$
\operatorname{amgu}_{x=t}(S)=S \backslash \operatorname{rel}(x, S) \backslash \operatorname{rel}(t, S) \cup(\operatorname{rel}(x, S) \uplus \operatorname{rel}(t, S))^{*}
$$

For the ensuing discussion, denote $\operatorname{rel}(x, S) \uplus \operatorname{rel}(t, S)$ by $S_{b}$. Rather than directly compute $S_{b}^{*}$ and use this closure to calculate $a m g u_{x=t}(S)$, we compute the partial sharing information $S \backslash \operatorname{rel}(x, S) \backslash \operatorname{rel}(t, S) \cup S_{b}$ and record information that enables $S_{b}^{*}$ to be computed on demand. The size of the partial sharing information inferred using this strategy is never more, and is often significantly 
less, than that inferred using full sharing. The key issue is to resolve which groups belong to $S_{b}$ so that we can recover $S_{b}^{*}$ on demand. One way to deduce $S_{b}$, and hence $S_{b}^{*}$, is to save the equation $x=t$ because if $G \in S \backslash \operatorname{rel}(x, S) \backslash \operatorname{rel}(t, S) \cup S_{b}$ then $G \in S_{b}$ iff $\operatorname{rel}(x=t, G) \neq \emptyset$. Since only variables in the equation actually matter in $\operatorname{rel}(x=t, G)$, it is sufficient to record the clique $\operatorname{var}(x=t)$ to recover $S_{b}$ and thereby derive full sharing. To summarise, the computational domain of Sharing is replaced with pairs of the form $\langle E, S\rangle \in C L \times$ Sharing where each clique in the first component is an abstraction of an equation and is used to recover the full sharing information that $\langle E, S\rangle$ describes. The following function formalises this idea since it maps each abstract values in $C L \times$ Sharing to the sharing abstractions they describe:

Definition 2. $\langle E, S\rangle \propto S^{\prime}$ iff $r(\langle E, S\rangle)=S^{\prime}$ where $r(\langle E, S\rangle)$ is defined as follows:

$$
\begin{gathered}
r(\langle\emptyset, S\rangle)=S \\
r\left(\left\langle\{e\} \cup E^{\prime}, S\right\rangle\right)=S^{\prime \prime} \cup \operatorname{rel}\left(e, S^{\prime \prime}\right)^{*}
\end{gathered}
$$

where $e \in E, E^{\prime}=E \backslash\{e\}$ and $S^{\prime \prime}=r\left(\left\langle E^{\prime}, S\right\rangle\right)$.

This function is called the recovery function. Although this recovery function calls closure under union operator, the recovery function is itself only called on demand. Henceforth, we abbreviate $r(\langle E, S\rangle)$ by $r(E, S)$.

Example 5. Let $\langle E, S\rangle=\left\langle\left\{e_{1}, e_{2}, e_{3}\right\}, S\right\rangle$, where $e_{1}=\{X, Y\}, e_{2}=\{Y, Z\}$, $e_{3}=\{Y, W\}$ and $S=\{\emptyset,\{X\},\{Y\},\{Z\},\{W\}\}$. Thus we can recover the full sharing information $S^{\prime}$ that $\langle E, S\rangle$ describes by unfolding definition 2 in the following fashion:

$$
\begin{gathered}
S^{\prime}=r\left(\left\{e_{1}, e_{2}, e_{3}\right\}, S\right)=r\left(\left\{e_{3}\right\}, r\left(\left\{e_{1}, e_{2}\right\}, S\right)\right)=r\left(\left\{e_{3}\right\}, r\left(\left\{e_{2}\right\}, r\left(\left\{e_{1}\right\}, S\right)\right)\right) \\
S_{1}=r\left(\left\{e_{1}\right\}, S\right)=S \cup \operatorname{rel}\left(e_{1}, S\right)^{*}=\{\emptyset,\{X\},\{Y\},\{Z\},\{W\},\{X, Y\}\} \\
S_{2}=r\left(\left\{e_{2}\right\}, S_{1}\right)=S_{1} \cup \operatorname{rel}\left(e_{2}, S_{1}\right)^{*}=\left\{\begin{array}{l}
\emptyset,\{X\},\{Y\},\{Z\},\{W\}, \\
\{X, Y\},\{Y, Z\},\{X, Y, Z\}
\end{array}\right\} \\
S^{\prime}=r\left(\left\{e_{3}\right\}, S_{2}\right)=S_{2} \cup \operatorname{rel}\left(e_{3}, S_{2}\right)^{*}=\left\{\begin{array}{l}
\emptyset,\{X\},\{Y\},\{Z\},\{W\},\{X, Y\}, \\
\{Y, Z\},\{X, Y, Z\},\{Y, W\}, \\
\{X, Y, W\},\{Y, Z, W\},\{X, Y, Z, W\}
\end{array}\right\}
\end{gathered}
$$

One natural question is whether the order in which cliques are considered in the recovery process actually matters. The following lemma is a partial answer to this question: it shows that the ordering does not matter when there are only two cliques in the first component.

\section{Lemma 1.}

$S \cup \operatorname{rel}\left(e_{1}, S\right)^{*} \cup \operatorname{rel}\left(e_{2}, S \cup \operatorname{rel}\left(e_{1}, S\right)^{*}\right)^{*}=S \cup \operatorname{rel}\left(e_{2}, S\right)^{*} \cup \operatorname{rel}\left(e_{1}, S \cup \operatorname{rel}\left(e_{2}, S\right)^{*}\right)^{*}$

The following proposition lifts this result to an arbitrary number of cliques; it also means that the recovery function satisfies the diamond property. 
Proposition 1. $r(E, S)=r(P, S)$ whenever $P$ is a permutation of $E$.

The abstract domain $C L \times$ Sharing is a non-canonical representation because different pairs $\left\langle E_{1}, S_{1}\right\rangle \neq\left\langle E_{2}, S_{2}\right\rangle$ can map to the same sharing abstraction during recovery. To obtain a partially ordered domain, it is necessary to induce an equivalence relation on $C L \times$ Sharing. We therefore define $\left\langle E_{1}, S_{1}\right\rangle \equiv\left\langle E_{2}, S_{2}\right\rangle$ iff $r\left(E_{1}, S_{1}\right)=r\left(E_{2}, S_{2}\right)$ and consider the abstract domain XSharing $=(C L \times$ Sharing $)_{\equiv}$, that is, the computational domain of sets of equivalence classes induced by $\equiv$. The partial ordering over XSharing, denoted by $\sqsubseteq$, can then by defined as $\left\langle E_{1}, S_{1}\right\rangle \sqsubseteq\left\langle E_{2}, S_{2}\right\rangle$ iff $r\left(E_{1}, S_{1}\right) \subseteq r\left(E_{2}, S_{2}\right)$. It then follows that $\langle X$ Sharing, $\sqsubseteq\rangle$ is a complete lattice.

For notational simplicity, we blur the distinction between elements of XSharing and the elements of $C L \times$ Sharing and interpret the pair $\left\langle E_{1}, S_{1}\right\rangle \in C L \times$ Sharing as representing the equivalence class $\left[\left\langle E_{1}, S_{1}\right\rangle\right]_{\equiv} \in X$ Sharing. For example, the recovery function over XSharing is the natural lifting of this function to the domain of equivalence classes.

\section{Abstract unification}

The rationale for our work is to avoid applying the calculating closure under union operator in abstract unification. In the following definition, we detail how to avoid the closures by instead creating cliques in the first component. An important consequence of this scheme, is that the operator is polynomial in the number of groups. In what follows, we prove that this new notion of abstract unification is both sound and precise.

\section{Definition 3.}

$$
\operatorname{xamgu}_{x=t}(\langle E, S\rangle)=\left\langle\begin{array}{c}
\max (E \cup\{\operatorname{var}(x=t)\}), \\
(S \backslash \operatorname{rel}(x=t, S)) \cup(\operatorname{rel}(x, S) \uplus \operatorname{rel}(t, S))
\end{array}\right\rangle
$$

Henceforth, we abbreviate $x a m g u_{x=t}(\langle E, S\rangle)$ by $\operatorname{xamgu}_{x=t}(E, S)$. Note that we only need to maintain maximal cliques in the first component because the effect of a smaller clique is subsumed by that of a larger one (one that strictly contains it). Therefore, we say a clique $e_{i}$ is redundant in $E \in C L$ if there exists $e_{j} \in E$ such that $e_{i} \subseteq e_{j}$. In that this is just one way of detecting redundancy: other more sophisticated ways are also possible.

Example 6. Consider $\langle E, S\rangle$ where $E=\{\{X, Y, Z\}\}$ and $S=\{\emptyset,\{X, Y\},\{X, Z\}$, $\{Z, W\}\}$. Then $\operatorname{xamgu}_{Y=Z}(E, S)=\langle\{\{X, Y, Z\}\},\{\emptyset,\{X, Y, Z\},\{X, Y, Z, W\}\}\rangle$

Lemma 2 explains how xamgu coincides with amgu when there is no clique in the first component.

Lemma 2. $r\left(x a m g u_{x=t}(\emptyset, S)\right)=\operatorname{amgu}_{x=t}(S)$.

Proposition 2 also builds towards the main correctness theorem by showing the equivalence of of two instances of abstract unification that operate on pairs whose clique sets differ by just one clique. 
Proposition 2. $r\left(\operatorname{xamgu}_{x=t}(E, S)\right)=r\left(\operatorname{xamgu}_{x=t}(E \backslash\{e\}, r(\{e\}, S))\right)$ where $e \in E$.

By applying proposition 2 inductively, it follows that $r\left(x a m g u_{x=t}(E, S)\right)=$ $r\left(x_{a m g u}=t(\emptyset, r(E, S))\right)$, that is, the result of applying xamgu to $\langle E, S\rangle$ coincides with the sharing abstraction obtained by applying xamgu to $\langle\emptyset, r(E, S)\rangle$. This result leads into Theorem 1 which states that xamgu neither compromises correctness not precision with respect to the classic approach of set-sharing.

Theorem 1. $r\left(x a m g u_{x=t}(E, S)\right)=\operatorname{amg}_{x=t}(r(E, S))$.

Example 7. Recall from example 6 that $\operatorname{xamgu}_{Y=Z}(E, S)=\langle\{\{X, Y, Z\}\}$, $\{\emptyset,\{X, Y, Z\},\{X, Y, Z, W\}\}\rangle$. By applying $r$ to the $\langle E, S\rangle$ pair we obtain $r(E, S)$ $=\{\emptyset,\{X, Y\},\{X, Z\},\{Z, W\},\{X, Y, Z\},\{X, Y, Z, W\},\{X, Z, W\}\}$. Then applying amgu to $r(E, S)$ yields amg $u_{Y=Z}(r(E, S))=\{\emptyset,\{X, Y, Z\},\{X, Y, Z, W\}\}$. Observe $r\left(\operatorname{xamgu}_{Y=Z}(E, S)\right)=\operatorname{amg}_{Y=Z}(r(E, S))$ as predicted by the theorem.

\section{Projection}

Projection on Sharing is relatively simple. In our proposal, projection is not straightforward as one might think. If projection was defined as $\langle E, S\rangle \uparrow \uparrow V=$ $\langle E\lceil V, S\lceil V\rangle$, then cliques would be possibly lost by projection which could affect recovery and can possibly compromise the soundness of the analysis as a whole. For example, suppose $\langle E, S\rangle=\langle\{\{X, Y\}\},\{\emptyset,\{Z, X\},\{Y, W\}\}\rangle$ and $V=\{Z, W\}$. Then $\langle E\lceil V, S \uparrow V\rangle=\langle\emptyset,\{\emptyset,\{Z\},\{W\}\}\rangle$ and hence we lose the information that $Z$ and $W$ could possibly share. In our projection operator, we divide the cliques into three classes. We need to apply a polynomial transformation for all cliques in the first class before applying the classical projection operator $\uparrow$. It is easy to do projection for the cliques in the second class. No projection is needed for the cliques in the third class.

\subsection{First Class}

Let $V \subseteq \mathcal{V}$ be a set of variables of interest. Every clique $e$ such that $e \cap V=\emptyset$ is in the first class and it will be totally projected away. Usually the first class cliques are generated by renaming. One way to keep the effect of a clique $e$ in the first class is applying the recovery function. This is precise but inefficient because of the closure under union operator in the recovery function. To avoid this, we maintain the effect of $e$ by replacing $e$ with a new clique $e^{\prime}$ that simulates the effect of $e$. This guarantees soundness but could lose precision. First, we define two auxiliary functions.

Observe that if we use $\cup \operatorname{rel}(e, S)$ to replace $e$, we will ensure $\operatorname{rel}(e, S)$ is selected because $\operatorname{rel}(e, S) \subseteq \operatorname{rel}(\cup \operatorname{rel}(e, S), S)$.

Definition 4. Function Replace $: C L \times$ Sharing $\rightarrow C L$ is defined by

$$
\operatorname{Replace}(E, S)=\max (\{\cup \operatorname{rel}(e, S) \mid e \in E\})
$$


We say that two cliques $e_{1}$ and $e_{2}$ are related with respect to a sharing abstraction $S$ if $\operatorname{rel}\left(e_{2}, \operatorname{rel}\left(e_{1}, S\right)\right) \neq \emptyset$. We also say $e_{i}$ and $e_{j}$ are related with respect to $S$ if $e_{i}$ is related to $e_{l}$ with respect to $S$ and $e_{l}$ is related to $e_{j}$ with respect to $S$. We say $E$ is related with respect to $S$ if every $e \in E$ is related to each other with respect to $S$.

Definition 5. Let $E_{1}$ be a set of cliques in the first class. We define a rewriting rule $\Longrightarrow_{S}$ by $E_{1} \Longrightarrow_{S} E_{2}$ if $e_{1}, e_{2} \in E_{1} \wedge \operatorname{rel}\left(e_{2}, \operatorname{rel}\left(e_{1}, S\right)\right) \neq \emptyset \wedge E_{2}=E_{1} \backslash$ $\left\{e_{1}, e_{2}\right\} \cup\left\{e_{1} \cup e_{2}\right\}$; otherwise $E_{1} \Longrightarrow_{S} E_{1}$.

We write $E \Longrightarrow{ }_{S}^{*} E^{\prime}$ if there exist $E_{1}, E_{2}, \ldots, E_{n}$ with $E_{1}=E, E_{n}=E^{\prime}$, $E_{i} \Longrightarrow_{S} E_{i+1}$ for $0 \leq i \leq n-1$ and $E^{\prime} \Longrightarrow_{S} E^{\prime}$. That is we apply $\Longrightarrow_{S}$ repeatedly until all the related cliques are combined into one. In other words, there are no two cliques that are related with respect to $S$ in $E^{\prime}$. Once we have $E^{\prime}$, we can apply the Replace function to replace all the cliques in $E^{\prime}$. Note that the order in which cliques are considered and combined does not effect the final result. Thus this definition satisfies the diamond property and is well-defined.

Let $E \Longrightarrow{ }_{S}^{*} E^{\prime}$. Then here exists $E_{1}, E_{2}, \ldots, E_{n}$ with $E_{1}=E, E_{n}=E^{\prime}$, $E_{i} \Longrightarrow_{S} E_{i+1}$ for $0 \leq i \leq n-1$. Note that $n$ is bounded by the number of groups. Each combination of two related cliques is polynomial. Thus the transformation is polynomial in the number of groups too.

Example 8. Let $E=\left\{\left\{X_{5}, X_{8}\right\},\left\{X_{5}, X_{6}\right\}\right\}, S=\left\{\emptyset,\left\{X_{1}, X_{6}, X_{8}\right\},\left\{X_{4}, X_{8}\right\}\right.$, $\left.\left\{X_{5}\right\},\left\{X_{2}, X_{6}\right\}\right\}$ and $V=\left\{X_{1}, X_{2}, X_{3}, X_{4}\right\}$. Then $E^{\prime}=\left\{\left\{X_{5}, X_{6}, X_{8}\right\}\right\}$ where $E \Longrightarrow{ }_{S}^{*} E^{\prime}$.

Every $e \in E$ is replaced by a corresponding $e^{\prime} \in \operatorname{Replace}\left(E^{\prime}, S\right)$ where $E \Longrightarrow_{S}^{*}$ $E^{\prime}$. There exists $e^{\prime \prime} \in E^{\prime}$ such that $e^{\prime}=\cup \operatorname{Urel}\left(e^{\prime \prime}, S\right)$. Observe that $e \subseteq e^{\prime \prime}$ follows from the definition of $\Longrightarrow_{S}^{*}$. Thus $\operatorname{rel}(e, S) \subseteq \operatorname{rel}\left(e^{\prime \prime}, S\right)$ and $\cup \operatorname{rel}(e, S) \subseteq$ $\cup \operatorname{rel}\left(e^{\prime \prime}, S\right)=e^{\prime}$. Thus $e^{\prime}$ actually includes the effect of $e$. If $e^{\prime}$ is not relevant to $V$ then it says nothing about $V$ and hence it does not matter at all. Therefore we can delete all the cliques that are not relevant to $V$ in $\operatorname{Replace}\left(E^{\prime}, S\right)$ where $E \Longrightarrow{ }_{S}^{*} E^{\prime}$.

Definition 6. Let $E$ be a set of cliques in the first class and $V$ is a set of variables. The function $T: C L \times$ Sharing $\rightarrow C L$ is defined by $T(E, S)=$ $\operatorname{Replace}\left(E^{\prime}, S\right) \backslash E^{\prime \prime}$ where $E \Longrightarrow{ }_{S}^{*} E^{\prime}, E^{\prime \prime}=\left\{e^{\prime} \in \operatorname{Replace}\left(E^{\prime}, S\right) \mid e^{\prime} \cap V=\emptyset\right\}$.

Note that $T(\emptyset, S)=\emptyset$.

Example 9. Continuing with example 8, observe that $\operatorname{Replace}\left(E^{\prime}, S\right)=\left\{\left\{X_{1}\right.\right.$, $\left.\left.X_{2}, X_{4}, X_{5}, X_{6}, X_{8}\right\}\right\}$ where $E \Longrightarrow_{S}^{*} E^{\prime}$. Hence Replace $\left(E^{\prime}, S\right) \backslash E^{\prime \prime}=\operatorname{Replace}\left(E^{\prime}, S\right)$ because $E^{\prime \prime}=\emptyset$. Thus, $T(E, S)=\left\{\left\{X_{1}, X_{2}, X_{4}, X_{5}, X_{6}, X_{8}\right\}\right\}$.

Proposition 3. (Soundness)

Let $E$ be a set of cliques in the first class and $E^{\prime}=T(E, S)$. Then

$$
r(E, S) \uparrow V \subseteq r\left(E^{\prime} \uparrow V, S \uparrow V\right)
$$




\subsection{Second Class and Third Class}

Let $V$ be a set of variables, $\langle E, S\rangle \in X$ Sharing and $e \in E$. If $e \cap V \neq \emptyset$ and $e \nsubseteq V$, we say that the clique $e$ is in the second class. If the clique $e$ is a subset of $V$ then we say it is in the third class.

Proposition 4. Let $V$ be a set of variables. If $\operatorname{rel}(e, S)=\operatorname{rel}(e \uparrow V, S)$ for each $e \in E$ then $r(E, S) \uparrow V=r(E \uparrow V, S \uparrow V)$.

Example 10. Let $\langle E, S\rangle=\langle\{\{X, Y\},\{Z, W\}\},\{\emptyset,\{X, Y, Z\},\{Z, W\}\}\rangle$ and $V=$ $\{X, Z\}$. Then $r(E, S)=\{\emptyset,\{X, Y, Z\},\{Z, W\},\{X, Y, Z, W\}\}$ and hence $r(E, S) \mid$ $V=\{\emptyset,\{X, Z\},\{Z\}\}$. On the other hand, $\langle E \uparrow V, S \uparrow V\rangle=\langle\{\{X\},\{Z\}\},\{\emptyset$, $\{X, Z\},\{Z\}\}\rangle$. Therefore $r(E, S) \uparrow V=r(E \uparrow V, S \uparrow V)$.

Lemma 3. If a clique $e$ is in second class then $\operatorname{rel}(e, S)=\operatorname{rel}(e \uparrow V, S)$.

According to lemma 3 and proposition 4, we can directly project those cliques in the second class without losing soundness and precision. It is obvious that $\operatorname{rel}(e, S)=\operatorname{rel}(e \uparrow V, S)$ is true for each clique in the third class. Note that the second class and the third class are both relevant to $V$ while the first class is not. Thus we can conclude that we can directly apply classical projection to all those cliques that are relevant to $V$. This is useful for implementation.

\subsection{Projection}

Therefore, we define the projection as following.

Definition 7. Let $\mathcal{V}$ be the set of all variables and $V \in \wp(\mathcal{V})$. The projection function $\uparrow \uparrow:$ XSharing $\times \wp(\mathcal{V}) \rightarrow$ XSharing is defined as $\langle E, S\rangle \uparrow \uparrow V=$ $\left\langle\left(T\left(E_{1}, S\right) \cup E_{2} \cup E_{3}\right)\lceil V, S \uparrow V\rangle\right.$ where $E_{1}$ contains the first class cliques in $E$, $E_{2}$ contains the second class cliques in $E$ and $E_{3}$ contains the third class cliques in $E$.

Theorem 2. (Soundness)

Let $V$ be a set of variables and $\langle E, S\rangle \in$ XSharing. Then $r(E, S) \uparrow V \subseteq$ $r(\langle E, S\rangle \uparrow V)$.

Although the projection operator needs to transform the first class cliques, it is still faster than applying the recovery function because $T$ is polynomial in the number of groups.

\section{Other Operations and Parameter Throughout}

The design of the analysis is completed by defining other operators that are required by an analysis engine. The initial state is $\langle\emptyset$, init $(\varepsilon)\rangle$ where $\operatorname{init}(\varepsilon)$ describes the empty substitution [18]. The renaming operator Rename $:$ XSharing $\rightarrow$ $X$ Sharing is defined by Rename $(\langle E, S\rangle)=\langle\operatorname{tag}(E), \operatorname{tag}(S)\rangle$ where $\operatorname{tag}$ is classical 
tagging function defined in [18]. The join operator $\sqcup:$ XSharing $\times X$ Sharing $\rightarrow$ $X$ Sharing is defined by $\left\langle E_{1}, S_{1}\right\rangle \sqcup\left\langle E_{2}, S_{2}\right\rangle=\left\langle E^{\prime}, S^{\prime}\right\rangle$ where $r\left(E^{\prime}, S^{\prime}\right)=r\left(E_{1}, S_{1}\right) \cup$ $r\left(E_{2}, S_{2}\right)$. In practice, we use $\left\langle E_{1}, S_{1}\right\rangle \bar{\sqcup}\left\langle E_{2}, S_{2}\right\rangle=\left\langle\max \left(E_{1} \cup E_{2}\right), S_{1} \cup S_{2}\right\rangle$ as an approximation. Observe $r\left(E_{1}, S_{1}\right) \cup r\left(E_{2}, S_{2}\right) \subseteq r\left(\max \left(E_{1} \cup E_{2}\right), S_{1} \cup S_{2}\right)$. Thus it is sound but may lose precision.

Example 11. Let $e_{1}=\{x, y\}, e_{2}=\{y, z\}, S_{1}=\{\emptyset,\{x, y\},\{z\}\}$ and $S_{2}=$ $\{\emptyset,\{y, z\},\{x\}\}$. Then $r\left(\left\{e_{1}\right\}, S_{1}\right) \cup r\left(\left\{e_{2}\right\}, S_{2}\right)=\{\emptyset,\{x\},\{z\},\{x, y\},\{y, z\}\}$ while $r\left(\left\{e_{1}, e_{2}\right\}, S_{1} \cup S_{2}\right)=\{\emptyset,\{x\},\{z\},\{x, y\},\{y, z\},\{x, y, z\}\}$. This is not precise because it says variables $x, y, z$ share a variable.

Basically, more cliques mean more efficiency and less cliques mean more precision. In fact, we can think Sharing is the most precise case of XSharing because it saves no clique in first component. We can control the complexity/precision ratio in two way:

- When do we create clique? The analysis is parameterised by a cost threshold $k$ : a clique is only created if the resulting number of sharing groups exceed a predefined threshold $k$.

- Which clique do we create or remove? A practical analyser requires a grounding operator whose effect is to ground given set of variables in a state. To this end, a grounding operator ground $: X$ Sharing $\times \mathcal{V} \rightarrow X$ Sharing is introducing that is defined by

$$
\operatorname{ground}(\langle E, S\rangle, x)=\langle E \backslash \operatorname{rel}(x, E), r(\operatorname{rel}(x, E), S) \backslash \operatorname{rel}(x, r(\operatorname{rel}(x, E), S))\rangle
$$

Observe that cliques are possibly removed from first component and that the overall size of the abstraction is not increased by this operator. This operator is applied to model the effect of a built-in predicate such as $>/ 2$. The effect of $>/ 2$ is to ground the variables associated with this operator. For instance, the effect of $X>Y$ is to ground both $X$ and $Y$.

\section{Implementation}

We have implemented the new abstract domain and incorporated it to a top down framework which is based on Nilsson [23]. The implementation is written in $\mathrm{C}++$. To obtain a credible experimental comparison, both the classical Sharing and XSharing are implemented using the same language, the same framework, the same data structure and the same primitive operators. Table 1 compares Sharing and XSharing on cost and precision using some standard benchmark programs. The first column and the fifth column list the names of programs. The second column, the third column, the sixth column and the seventh column are time performance for XSharing and Sharing respectively. The fourth and the last column are the loss of precision in percentage considering pair sharing information. Suppose there are $n$ program points in a program. For program point $i$, the pair sharing information computed by Sharing is $P S_{i}$ while XSharing 
computes $X P S_{i}$. Pair-sharing $P S_{i}$ must be a subset of $X P S_{i}$ since $X$ Sharing is approximate. It follows that $\left|X P S_{i}\right| \geq\left|P S_{i}\right|$. Then pair sharing precision lost at program point $i$ can be defined by $\left(\left|X P S_{i}\right|-\left|P S_{i}\right|\right) /\left|P S_{i}\right|$. The pair sharing precision lost for whole program is $\sum_{i=1}^{n}\left(\left(\left|X P S_{i}\right|-\left|P S_{i}\right|\right) /\left|P S_{i}\right|\right) / n$.

Benchmarks are divided into two groups in table 1. XSharing and Sharing are both acceptable for the programs in the first group. For the programs in the second group, XSharing is much faster. Classical Sharing takes hundreds seconds for peepl.pl, sdda.pl, ga.pl and read.pl while XSharing only takes several seconds. This is not a surprise because all key operators are polynomial.

Table 1. Time Performance Considering Effects of Built-in Predicates; $k=0$

\begin{tabular}{|l|l|r|r|l|l|l|l|}
\hline \multicolumn{7}{|c|}{ OPU: Intel(R) Pentium(R) 4 CPU 2.40GHz. } \\
Operating System: Windows XP \\
\hline & XSharing & Sharing & Precision & & XSharing & Sharing & Precision \\
\hline Program & Time & Time & Pair lose & Program & Time & Time & Pair lose \\
\hline append.pl & 0.008 & 0.016 & 0 & qsort.pl & 0.008 & 0.015 & 0 \\
\hline merge.pl & 0.015 & 0.016 & 0 & path.pl & 0.008 & 0.008 & 0 \\
\hline zebra.pl & 0.031 & 0.016 & 0 & life.pl & 0.016 & 0.041 & 0 \\
\hline disj_r.pl & 0.015 & 0.015 & 0 & queens.pl & 0.008 & 0.047 & 0 \\
\hline browse.pl & 0.032 & 0.047 & 0 & gabriel.pl & 0.016 & 0.031 & 0 \\
\hline treesort.pl & 0.015 & 0.172 & 0.5 & dnf.pl & 0.11 & 0.062 & 11 \\
\hline boyer.pl & 0.047 & 0.062 & 0 & tsp.pl & 0.047 & 0.032 & 5 \\
\hline peephole.pl & 0.078 & 0.125 & 0 & kalah.pl & 0.047 & 0.281 & 0 \\
\hline aiakl.pl & 0.094 & 0.468 & 3 & treeorder.pl & 0.14 & 1.172 & 0 \\
\hline \hline cs_r.pl & 0.078 & 1.187 & 0 & boyer.pl & 1.547 & 7.86 & 0 \\
\hline peep.pl & 1.86 & 137.797 & 0 & read.pl & 2.469 & 129.859 & 0 \\
\hline sdda.pl & 5.453 & 151.68 & 0 & ga.pl & 0.281 & 209.687 & 0 \\
\hline
\end{tabular}

There are 24 programs in table 1 . The pair sharing information computed by XSharing is exactly same as Sharing for 20 programs. For the programs peepl.pl, sdda.pl, ga.pl and read.pl, the performance is remarkably improved while we have exactly same pair sharing information. There is loss of precision for 4 programs. This is caused by ordering, join and projection operators. For program treesort.pl, the precision loss is $0.5 \%$ that is almost equal to zero. An interesting program is dnf.pl. We lost $11 \%$ precision for this program while Sharing is faster than XSharing. This is a negative result for our analysis. We investigated this program and found out that there are many variables that are ground and thus closure under union operations are applied on small sets. This explains why Sharing is actually faster. Therefore, in our opinion, our analysis is more suitable for complex programs that involve many variables and closure under union operations.

We can adjust the threshold $k$ to control the complexity/precision ratio of the system. The threshold is 0 for all programs in table 1 and there are four programs that lose precision. We can find the minimal $k$ that guarantees these 
programs do not lose precision. The minimal $k$ are listed in the last column in table 2 . The other columns are same as table 1 .

Table 2. The Minimal Threshold without Losing Precision

\begin{tabular}{|l|l|l|l|c|}
\hline & XSharing & Sharing & Precision & Threshold \\
\hline Program & Time & Time & Pair lose & $\mathrm{k}$ \\
\hline treesort.pl & 0.328 & 0.172 & 0 & 16 \\
\hline dnf.pl & 0.047 & 0.062 & 0 & 16 \\
\hline aiakl.pl & 0.483 & 0.468 & 0 & 171 \\
\hline tsp.pl & 0.020 & 0.032 & 0 & 23 \\
\hline
\end{tabular}

There are many built-in predicates in a real program and it is important to consider the effect of them. Thus we have supported all built-in predicates in the benchmark programs. For example, we have considered built-in predicates such as $=/ 2$ and sort $/ 2$ whose effect is to apply abstract unification. We also processed built-in predicates such as $>/ 2$ and ground/1 whose effect is to ground variables. Other control built-in predicates such as ; $/ 2$ and $\rightarrow / 2$ are also processed. Note that considering the effect of built-in predicates with grounding effect can improve the efficiency significantly because many groups are removed.

\section{Related work}

There have been much research in sharing analysis of logic programs $[17,24,4$, $20,8,19,9,22,14,5]$ that have attempted to tame the computational aspects of this domain without compromising too much precision. One approach is reducing the number of groups that can possibly arise is due to Fecht [12] and Zaffanella et al. [25] who use maximal elements to represent downward closed powersets of variables. In fact Zaffanella et al. [25] apply a pair of sharing abstractions, which is almost same as our proposal. The key difference is interpretation. In [25], a clique represents all sharing groups that contained within it. In this paper, a clique is used to select the basis of a closure.

Bagnara et al. [3] also argue that Sharing is redundant for pair-sharing based on the following assumption: that the goal of sharing analysis for logic program is to detect which pairs of variables are definitely independent. A group $G$ is redundant with respect to a sharing abstraction $S$ if $S$ already contains the pair sharing information that $G$ contains. Thus they obtain a simpler domain and, perhaps more importantly, reduce complexity of abstract unification. That is, closure under union is not needed. This simpler domain is as precise as Sharing on pair-sharing. On the other hand, Bueno et al. [6] argue that the assumption in [3] is not always valid. First, there are applications that use Sharing other than retrieving sharing information between pairs of variables. Second, Sharing is more precise when combined with other source of information. In this paper, we consider a group is redundant if it can be regenerated using the cliques saved in first component. Moreover, the redundant concept is applied to cliques. 
Howe and King [16] present three optimisations for Sharing. These optimisations can be used when combine Sharing with freeness and linearity. One principle in [16] is that the number of sharing groups should be minimised. This principle is also used in our approach, though we do not seek to exploit either freeness or linearity.

Another thread of work is in the use different encodings of the Sharing domain. In [8], Codish et al. show that Sharing is isomorphic to the domain of positive Boolean functions [1]. The closure under union operator over Sharing corresponds to downward closure operator $\downarrow$ over Pos that maps a positive Boolean function to a definite Boolean function. A definite Boolean function thus corresponds to a sharing that is closed under union. This domain is efficient because of efficient data structure of Boolean function. Howe et al. propose a downward closure operator $\downarrow$ in [15]. Codish et al. [7] also propose an algebraic approach to the sharing analysis using set logic programs. This domain is isomorphic to Sharing and leads to intuitive definitions for abstract operators.

\section{Conclusion}

This paper presents a new domain for sharing analysis based on a new efficient representation for Sharing. All key operators are polynomial with respect to the number of groups and soundness proofs are given. Moreover, by remembering the cliques, we provide a scheme to reason about the relationship between cliques such as finding redundant cliques. Lastly, there is a parameter to control the complexity/precision ratio of the system. For the future work, we will devote more efforts on the precision and find more redundant cliques. It looks promising to explore how a pair can be recovered to non-redundancy sharing [3] by changing the representation of first component too.

\section{References}

1. Tania Armstrong, Kim Marriott, Peter Schachte, and Harald Søndergaard. Two Classes of Boolean Functions for Dependency Analysis. Science of Computer Programming, 31(1):3-45, 1998.

2. Roberto Bagnara, Roberta Gori, Patricia M. Hill, and Enea Zaffanella. Finite-Tree Analysis for Constraint Logic-Based Languages. Information and Computation, 193(2):84-116, 2004.

3. Roberto Bagnara, Patricia M. Hill, and Enea Zaffanella. Set-Sharing is Redundant for Pair-Sharing. Theoretical Computer Science, 277(1-2):3-46, 2002.

4. Roberto Bagnara, Enea Zaffanella, and Patricia M. Hill. Enhanced Sharing Analysis Techniques: A Comprehensive Evaluation. Theory and Practice of Logic Programming, 5(1\&2):1-43, 2005.

5. Maurice Bruynooghe, Bart Demoen, Dmitri Boulanger, Marc Denecker, and Anne Mulkers. A Freeness and Sharing Analysis of Logic Programs Based on A Preinterpretation. In Radhia Cousot and David A. Schmidt, editors, Proceedings of the Third International Symposium on Static Analysis, volume 1145 of Lecture Notes in Computer Science, pages 128-142, London, UK, 1996. Springer-Verlag. 
6. Francisco Bueno and Maria J. García de la Banda. Set-Sharing Is Not Always Redundant for Pair-Sharing. In Yukiyoshi Kameyama and Peter J. Stuckey, editors, Proceedings of 7th International Symposium on Functional and Logic Programming, volume 2998 of Lecture Notes in Computer Science, pages 117-131. Springer, 2004.

7. Michael Codish, Vitaly Lagoon, and Francisco Bueno. An Algebraic Approach to Sharing Analysis of Logic Programs. Journal of Logic Programming, 42(2):111-149, 2000.

8. Michael Codish, Harald Søndergaard, and Peter J. Stuckey. Sharing and Groundness Dependencies in Logic Programs. ACM Transactions on Programming Languages and Systems, 21(5):948-976, 1999.

9. Agostino Cortesi and Gilberto Filé. Sharing Is Optimal. Journal of Logic Programming, 38(3):371-386, 1999.

10. Lobel Crnogorac, Andrew D. Kelly, and Harald Sondergaard. A Comparison of Three Occur-Check Analysers. In Radhia Cousot and David A. Schmidt, editors, Proceedings of Third International Symposium on Static Analysis, volume 1145 of Lecture Notes in Computer Science, pages 159-173. Springer, 1996.

11. Jacques Chassin de Kergommeaux and Philippe Codognet. Parallel Logic Programming Systems. ACM Computing Surveys, 26(3):295-336, 1994.

12. Christian Fecht. An Efficient and Precise Sharing Domain for Logic Programs. In Herbert Kuchen and S. Doaitse Swierstra, editors, Proceedings of the 8th International Symposium on Programming Languages: Implementations, Logics, and Programs, volume 1140 of Lecture Notes in Computer Science, pages 469-470. Springer, 1996.

13. Patricia M. Hill, Roberto Bagnara, and Enea Zaffanella. Soundness, Idempotence and Commutativity of Set-Sharing. Theory and Practice of Logic Programming, 2(2):155-201, 2002.

14. Patricia M. Hill, Enea Zaffanella, and Roberto Bagnara. A Correct, Precise and Efficient Integration of Set-Sharing, Freeness and Linearity for The Analysis of Finite and Rational Tree Languages. Theory and Practice of Logic Programming, 4(3):289-323, 2004.

15. J. M. Howe and A. King. Efficient Groundness Analysis in Prolog. Theory and Practice of Logic Programming, 3(1):95-124, January 2003.

16. Jacob M. Howe and Andy King. Three Optimisations for Sharing. Theory and Practice of Logic Programming, 3(2):243-257, 2003.

17. Dean Jacobs and Anno Langen. Accurate and Efficient Approximation of Variable Aliasing in Logic Programs. In Ross A. Overbeek Ewing L. Lusk, editor, Proceedings of the North American Conference on Logic Programming, pages 154-165. MIT Press, 1989.

18. Dean Jacobs and Anno Langen. Static Analysis of Logic Programs for Independent And-Parallelism. Journal of Logic Programming, 13(2\&3):291-314, 1992.

19. Andy King. Pair-Sharing over Rational Trees. Journal of Logic Programming, 46(1-2):139-155, 2000.

20. Vitaly Lagoon and Peter J. Stuckey. Precise Pair-Sharing Analysis of Logic Programs. In Proceedings of the 4th ACM SIGPLAN International Conference on Principles and Practice of Declarative Programming, pages 99-108. ACM Press, 2002.

21. Anno Langen. Advanced Techniques for Approximating Variables Aliasing in Logic Programs. PhD thesis, 1991.

22. Kalyan Muthukumar and Manuel V. Hermenegildo. Compile-Time Derivation of Variable Dependency Using Abstract Interpretation. Journal of Logic Programming, 13(2-3):315-347, 1992. 
23. Ulf Nilsson. Towards a Framework for the Abstract Interpretation of Logic Programs. In Pierre Deransart, Bernard Lorho, and Jan Maluszynski, editors, Proceedings of the Programming Language Implementation and Logic Programming, volume 348 of Lecture Notes in Computer Science, pages 68-82. Springer, 1989.

24. Harald Søndergaard. An Application of Abstract Interpretation of Logic Programs: Occur Check Reduction. In Bernard Robinet and Reinhard Wilhelm, editors, Proceedings of the 1st European Symposium on Programming, volume 213 of Lecture Notes in Computer Science, pages 327-338. Springer, 1986.

25. Enea Zaffanella, Roberto Bagnara, and Patricia M. Hill. Widening Sharing. In Gopalan Nadathur, editor, Proceedings of the International Conference on Principles and Practice of Declarative Programming, volume 1702 of Lecture Notes in Computer Science, pages 414-432, London, UK, 1999. Springer-Verlag. 